\title{
Mucosal versus muscle pain sensitivity in provoked vestibulodynia
}

This article was published in the following Dove Press journal:

Journal of Pain Research

12 August 2015

Number of times this article has been viewed

\author{
Kathryn Witzeman' \\ Ruby HN Nguyen ${ }^{2}$ \\ Alisa Eanes ${ }^{3}$ \\ Sawsan As-Sanie ${ }^{4}$ \\ Denniz Zolnoun ${ }^{5}$ \\ 'Department of Obstetrics and \\ Gynecology, Denver Health Medical \\ Center, Denver, CO, ${ }^{2}$ Division of \\ Epidemiology and Community Health, \\ University of Minnesota, Minneapolis, \\ MN, ${ }^{3}$ Pelvic Pain Research Unit, \\ Division of Advanced Laparoscopy \\ and Pelvic Pain, Department of \\ Obstetrics and Gynecology, University \\ of North Carolina School of Medicine, \\ Chapel Hill, NC, ${ }^{4}$ Department of \\ Obstetrics and Gynecology, Division \\ of Minimally Invasive Gynecologic \\ Surgery, University of Michigan, Ann \\ Arbor, MI, ${ }^{5}$ Department of Obstetrics \\ and Gynecology and Center for \\ Neurosensory Disorders, University \\ of North Carolina, Chapel Hill, \\ NC, USA
}

Background: An estimated $8.3 \%-16 \%$ of women experience vulvovaginal discomfort during their lifetime. Frequently these patients report provoked pain on contact or with attempted intercourse, commonly referred to as provoked vestibulodynia (PVD). Despite the burden of this condition, little is known about its potential etiologies including pelvic floor muscular dysfunction and mucosal components. This knowledge would be beneficial in developing targeted therapies including physical therapy.

Objective: To explore the relative contribution of mucosal versus muscle pain sensitivity on pain report from intercourse among women with PVD.

Design: In this proof of concept study, 54 women with PVD underwent a structured examination assessing mucosal and pelvic muscle sensitivity.

Methods: We examined three mucosal sites in the upper and lower vestibule. Patients were asked to rate their pain on cotton swab palpation of the mucosa using a 10-point visual analog scale. Muscle pain was assessed using transvaginal application of pressure on right and left puborectalis, and the perineal muscle complex. The Gracely pain scale $(0-100)$ was used to assess the severity of pain with intercourse, with women rating the lowest, average, and highest pain levels; a 100 rating the highest level of pain.

Results: The lower vestibule's mucosa 5.81 (standard deviation $=2.83$ ) was significantly more sensitive than the upper vestibule 2.52 (standard deviation $=2.6)(P<0.01)$ on exam. However, mucosal sensitivity was not associated with intercourse pain, while muscle sensitivity was moderately associated with both average and highest intensity of intercourse pain $(r=-0.46$, $P=0.01$ and $r=-0.42, P=0.02$ ), respectively.

Conclusion: This preliminary study suggests that mucosal measures alone may not sufficiently capture the spectrum of clinical pain report in women with PVD, which is consistent with the empirical success of physical therapy in this population.

Keywords: vulvodynia, provoked vestibulodynia, pain sensitivity, pelvic floor muscle pain, vulvar pain, pressure pain threshold, dyspareunia

\section{Introduction}

In a medical landscape filled with complex chronic pain disorders, none is as overlooked as pain generated in the female reproductive system. An estimated $8.3 \%-16 \%$ of women experience vulvovaginal discomfort at some point in their lifetime. ${ }^{1,2}$ Among those who seek medical care, up to $60 \%$ will consult more than three physicians prior to receiving an appropriate diagnosis. ${ }^{1,2}$ The most common complaint among these women is provoked vulvar pain on contact, vaginal penetration or attempted intercourse, referred to as provoked vestibulodynia (PVD). ${ }^{1-4}$
Correspondence: Kathryn Witzeman Department of Obstetrics and Gynecology, Denver Health Medica Center, 777 Bannock Street, MC 0660,

Denver, CO 80204, USA

Tel +l 3036029725

Fax + I 3036029734

Email kathryn.witzeman@dhha.org 
The earliest documentation of this condition dates back to the $1800 \mathrm{~s} .{ }^{5}$ Current literature primarily refers to criteria developed in Friedrich's study of 86 patients published in 1987. Friedrich coined the term "vulvar vestibulitis syndrome" based on constellation of three signs and symptoms: 1) severe pain on vestibular touch and entry dyspareunia, 2) tenderness to pressure within the vestibule, and 3) physical findings limited to erythema without other obvious pathology. ${ }^{6,7}$ Despite considerable subsequent controversy surrounding nomenclature, the diagnosis by gynecologists of this condition continues to be based on Friedrich's description of mucosal sensitivity. However, emerging literature suggests that mucosal sensitivity alone may not sufficiently capture the heterogeneity of patients with PVD. ${ }^{8}$ For example, in addition to mucosal sensitivity, a significant proportion of women with PVD experience pain in underlying pelvic musculature along with difficulty with volitional control of these muscles during clinical examination..$^{9-11}$ However, the extent to which abnormalities in the anatomical form or function of pelvic musculature may contribute to the experience of pain in normal activities of women (ie, sexual activity), remains wholly unknown. Additionally, further elucidation is needed of the potential interrelationship between how increased mucosal sensitivity may affect underlying musculature leading to compensatory contracture and hypertonicity which may further impact pain. Or conversely, underlying pelvic floor musculature dysfunction may primarily and more directly lead to a neurogenic referred mucosal sensitivity. These theories have yet to be fully substantiated in the literature at the foundational level. The utility of such findings directly relate to the ability of clinicians to target therapies to either (or both) causes of vulvar pain, mucosal or muscle. Current physical therapy regimens for PVD have been somewhat successful, ${ }^{12,13}$ however, these therapeutic regimens are diverse including soft tissue manipulation, myofascial release, core strengthening exercises, dry needling, etc and could be better targeted to the type of pain..$^{14,15}$ Furthermore, the ability to better subtype pain contributors would be particularly helpful if the patient is in need of multimodal treatment inclusive of pharmacotherapy and behavioral health modalities. ${ }^{16,17}$ Therefore, the purpose of this study is to investigate the relationship between pain reported during sexual intercourse (dyspareunia) and the measurement of mucosal and muscle pain sensitivity among women with PVD during a structured clinical exam. In this study, we used a modified version of a previously developed clinical methodology to examine in detail the relationship between a patient's self-report of pain during intercourse and mucosal and muscle pain sensitivity from a structured clinical research exam. ${ }^{18}$

\section{Methods}

This proof of concept pilot study was nested in a previously described study of women with PVD who in addition to completing a battery of questionnaires at two time points underwent a structured pelvic examination at the University of North Carolina (UNC). ${ }^{19}$ The UNC pelvic pain clinic's referral pattern consists of $82 \%$ clinician referred female patients, with a median pain duration of 5 years, as well as a median number of three physicians seen prior to their initial UNC pelvic pain clinic visit. As a whole, our cohort of women consisted of the most intractable spectrum of women with PVD. The subjects presented with a wide spectrum of both mucosal and muscular pain sensitivity assessed via previously described standardized methodology. ${ }^{18}$ Informed consent was obtained from all women who participated in this study. The Institutional Review Board at UNC, Chapel Hill, approved this study.

Of the previously described cohort of $137,{ }^{19} 54$ women had a structured research exam on their first clinic visit and met the inclusion criteria described below. Following the conventional gynecological evaluation and diagnosis of PVD, participants underwent a more nuanced mucosal and pelvic muscle assessment consistent with clinical practice. The diagnosis of PVD was made based on the subjective report of pain during intercourse and tenderness to touch during a cotton swab exam; the diagnosis was rendered after excluding other identifiable etiologies. Patients with co-morbid urogenital pain conditions (eg, vaginismus, generalized vulvodynia, interstitial cystitis), dermatological conditions (eg, lichen sclerosis), chronic pelvic pain defined as non-menstrual daily pain localized to the pelvic region, and neuropathies (eg, pudendal neuralgia) were excluded.

The clinical exam and history were abstracted from the review of the medical records. Two independent reviewers ${ }^{20}$ classified patients into clinically accepted subtypes of PVD: primary versus secondary. Women who developed PVD after a period of pain-free intercourse are considered secondary; those who endorse pain since coital debut or first tampon insertion are considered primary. The reviewers were masked to the participants' questionnaire response. Agreement between the two reviewers was high. Of the 54 participants, 20 were classified as having primary PVD subtype. The remaining 34 were either classified as having a secondary subtype $(n=32)$, defined as developing pain after a pain-free interval, or could not be classified $(n=2)$ due to missing or conflicting data. 
Our structured mucosal sensitivity assessment consisted of a standardized approach to palpation and localization of individual mucosal sites on the vestibular surface area. The upper vestibular sites were examined first followed by examination of the lower vestibule. The six anatomical sites (three in the anterior and three in the posterior vestibule) were determined with reference to the conventional "clock face". Using an imaginary clock, a total of 12 equidistant sites on the vestibule can be described circumferentially, with 12 and 6 positions corresponding to the anterior and posterior position on the midline (in dorsal lithotomy position). Thus, sites 2, 10, and 12 are located in the upper vestibule and sites 5, 6, and 7 are located in the lower vestibule (Figure 1). ${ }^{18}$ Furthermore, we used anatomical landmarks to standardize the location of these vestibular sites among patients. Sites 2 and 10 correspond to mid-position on the vestibular mucosa located on an imaginary line through the urethral meatus and parallel to the horizontal plane. Site 12 was located equidistant from the glans clitoris and the urethral meatus (measured vertically). The lower three sites

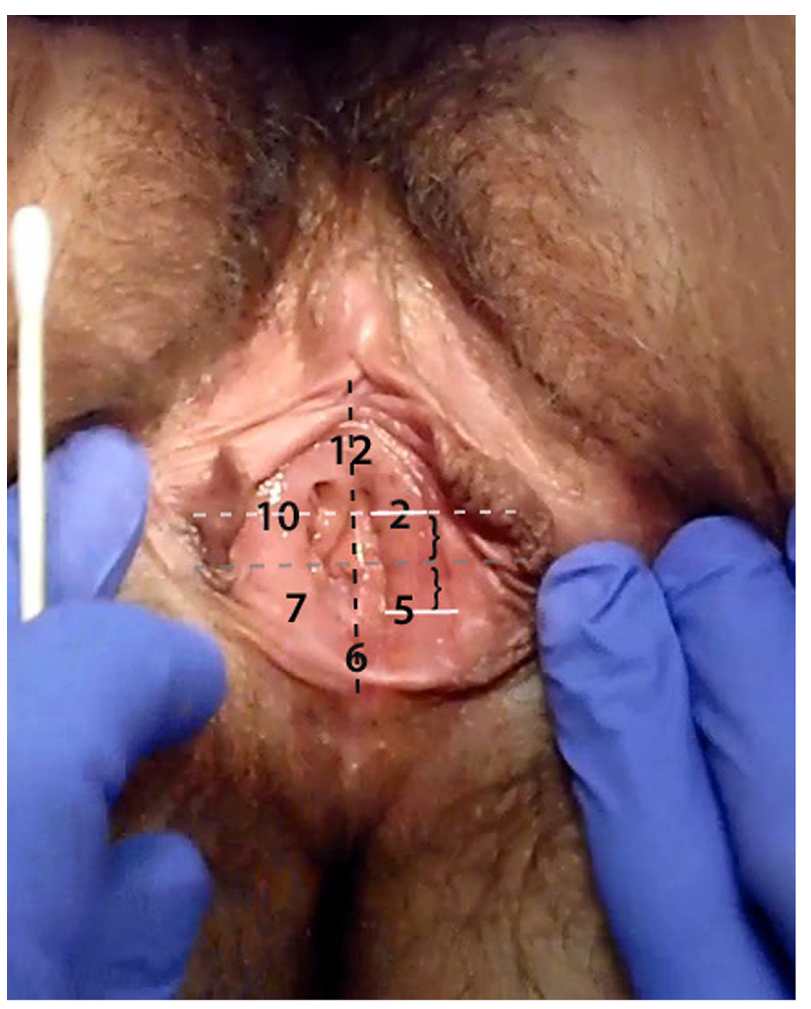

Figure I Anatomical locations of the mucosal sites tested with a cotton swab. Notes: Upper vestibule includes sites 2, 10, and 12. With 2 and 10 marking an imaginary line through the urethral meatus, and 12 is equidistant between. The lower vestibule sites include 5, 6, and 7 and are a mirror image of the upper vestibule. Reprinted from The Journal of Pain; 13(9); Zolnoun D, Bair E, Essick G, Gracely R, Goyal V, Maixner W; Reliability and reproducibility of novel methodology for assessment of pressure pain sensitivity in pelvis; pages 910-920; Copyright 2012, with permission from Elsevier. ${ }^{18}$ were selected to reflect the mirror image of the upper vestibular sites. Thus, 6 o'clock is the most posterior position on the vestibule and equidistant from 5 o'clock position on the right (subject's left) and 7 o'clock position on the left (subject's right).

With an inter-stimuli interval of 2 seconds, the upper and then lower vestibular sites were palpated in the following sequence: right, left, and midline. For example, examination of the upper vestibule consisted of palpating sites 10, 2, and 12 sequentially. Using a cotton swab, we applied an approximate force of 1 Newton to each of the six vestibular sites using a method modified from our previous work. ${ }^{18}$ The algometer was not used in this protocol since data from our group suggest that the inter-rater reliability of cotton swab testing with and without the use of the algometer is Kappa $=0.85$ (unpublished data). To this end, each site was palpated for an approximate duration of 1 second or until mucosal blanching was observed. Based on our experience using a nuanced algometer, ${ }^{18}$ the above-mentioned methodology of mucosal assessment corresponded to 1-1.5 Newton per vestibular site.

Following each palpation, participants were instructed to verbally report the severity of pain (pain threshold) on a Likert scale of 0 (no pain) to 10 (worst pain imaginable). A numerical average of the three vestibular sites was used to calculate the corresponding upper and lower vestibule's pressure pain threshold (PPT) for the individual participants.

Pelvic muscle PPT measurements were obtained using a modified Jaeger's technique, which consisted of digital calibration of the pressure with an algometer (Figure 2). ${ }^{21}$ For these preliminary data only, the pelvic muscle PPT was assessed using the index finger calibrated to apply pressure at an increment of $500 \mathrm{~g} / \mathrm{s}$ transvaginally, while carefully avoiding the sensitive vestibular mucosa. This methodology was adapted and validated in the pelvic musculature ${ }^{18}$ from the literature in orofacial pain and fibromyalgia where pressures as low as $1,000 \mathrm{~g}$ (in orofacial region), and as high as 4,000 $\mathrm{g}$ are commonly applied for assessment of muscle pain. ${ }^{22,23}$

Based on our experience using a nuanced algometer, ${ }^{18}$ the application of the above-mentioned methodology to the pelvic region leads to maximal stretch (ceiling effect) in pelvic musculature (specifically puborectalis) that varies from $500 \mathrm{~g}$ to upward of 3,000 g. Three muscle groups were examined including the perineal muscle complex (this includes the convergence of the posterior fibers of the bilateral bulbocavernosus muscles and the central midline of the transverse perineal muscle at 6 o'clock), the right puborectalis and the left puborectalis muscles (Figure 3). ${ }^{18}$ Pressure application points on the puborectalis were identified in the 


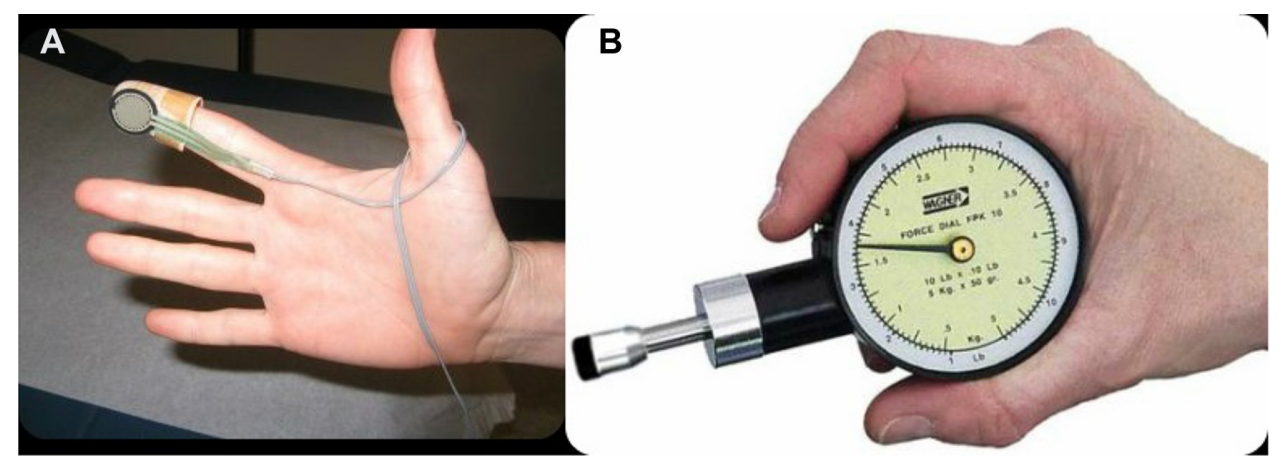

Figure 2 Finger sensor $(\mathbf{A})$ and algometer $(\mathbf{B})$ for calibration.

Notes: (A) Adapted from The Journal of Pain; 13(9); Zolnoun D, Bair E, Essick G, Gracely R, Goyal V, Maixner W; Reliability and reproducibility of novel methodology for assessment of pressure pain sensitivity in pelvis; pages 910-920; Copyright 2012, with permission from Elsevier. ${ }^{18}$

following manner. The ventral surface of the right index finger facing the subject's right pubic rami was carefully moved intravaginally. With a bent index finger (approximately 30-60 degrees at the interphalangeal joints), the examiner palpated the mid-pubic rami. This bony landmark allows us to identify the mid-segment (belly) of puborectalis muscle. Once this location was confirmed, the examiner palpated the puborectalis muscle, immediately adjacent/behind the pubic rami $(<0.5 \mathrm{~cm})$. Subsequently, the examiner rotated the index finger downward (approximately $1-1.5 \mathrm{~cm}$ ) parallel to the length of puborectalis. This was the point of pressure application for the assessment of PPT on the right and left puborectalis muscle. Following the assessment of the right side, the examiner rotated his/her right hand in order to palpate the left puborectalis as described above. ${ }^{18}$

Participants were instructed to verbally signal at the first sensation of pain at which point the pressure application was terminated by the examiner. The pressure at which the examiner stopped the pressure application was defined as the PPT and recorded by the examiner. Each muscle site was examined three times with an inter-stimuli interval of 15 seconds. Muscle PPT in each site was determined by the numerical average of these three measures. As with the mucosal data, the participant response data were analyzed based upon anatomic pain sensitivity as well as primary versus secondary pathology groupings.

Self-reported pain with intercourse was assessed by administering the Gracely pain scale, which asks women to rate the lowest, average, and maximal pain with intercourse on a scale of $0-100 .{ }^{24}$ Modified versions of this questionnaire are commonly used in assessing pain among patients with idiopathic pain disorders (eg, temporomandibular disorder and fibromyalgia).

Statistical analyses were performed using SAS 9.1 (SAS Institute Inc., Cary, NC, USA). Descriptive statistics (mean and standard deviation [SD] for continuous variables and frequency and percentages for bivariate variables) were calculated. Student's $t$-tests were used to compare continuous variables. We used a composite score consisting of the numerical average of the three vestibular sites in the lower and upper vestibule, respectively. We similarly averaged three threshold measures in order to obtain the site-specific muscle PPT. Pearson's correlation was used for the purposes of describing the correlation between subjective report of pain with intercourse and mucosal and muscle pain sensitivity respectively; the correlations were similarly examined in subgroups of women with PVD. Statistical significance was set to $(P<0.05)$ for all comparisons.

\section{Results}

Only $2 \%$ of eligible subjects declined participation in the study and were excluded in subgroup analysis. Our participants primarily consisted of college educated ( $80 \%)$, married (87\%), white women (84\%), aged 31 years ( $\mathrm{SD}=7$ ); $81 \%$ were nulliparous. The clinical subtype distribution of the study population included $37 \%$ classified as primary $(n=20)$ and $60 \%$ classified as secondary $(n=32)$, leaving two subjects classified as unknown.

On a pain scale of $0-10$, the average mucosal assessment during cotton swab testing of the lower vestibule 5.81 (SD $=2.83$ ) demonstrated more sensitivity than the upper vestibule $2.52(\mathrm{SD}=2.6)(P=0.01)$. Women presenting with primary and secondary PVD did not differ in severity of mucosal sensitivity on exam. However, self-reported severity of pain with intercourse showed some evidence of variation by subgroups of women with PVD, though these associations may be of marginal statistical significance. For instance, maximal intercourse-related pain was reported as $87.1(\mathrm{SD}=10.8)$ $(0-100$ scale) for women in the primary PVD subgroup and 72.9 ( $\mathrm{SD}=24.8)$ for women with secondary PVD $(P=0.06)$.

Pain scores elicited in the more sensitive lower vestibule $(0-10$ scale) by the cotton swab examination demonstrated 
a correlation with components of the modified Gracely pain scale (0-100) for the entire cohort of patients. However, while the average pain with intercourse was related to physical examination pain $(r=0.38, P<0.01)$, neither the lowest pain nor maximal pain with intercourse demonstrated correlation with pain on physical examination ( $r=0.24$ and 0.19 , respectively, $P<0.10$ for both). The above observation was true for both primary ( $r=0.27, P=0.29)$ and secondary subgroups ( $r=0.16, P=0.39)$.

Muscle pain threshold at each of the three tested sites varied from 500 to 2,000 $\mathrm{g}$ among women with PVD $(\mathrm{n}=30)$. In each site the three values used to define threshold were within 200-300 g of one another. The lowest PPT (highest tenderness) was observed in the perineal muscle complex with some women reporting pain at pressures as low as 500 g. While puborectalis was similarly more likely to be tender in patients, few women $(n=4)$ reported pain in the puborectalis muscles at pressures as high as those we observed in healthy controls (eg, 2,000 g).

We then used the PPT from the perineal complex to assess the relation between clinical pain report with sexual intercourse and muscle tenderness. Women with greater muscle tenderness (lower PPT) reported more pain during intercourse. Average and highest intercourse-related pain was significantly correlated with lower muscle PPTs on examination $(r=-0.46$, $P=0.01$ and $r=-0.42, P=0.02$, respectively) (Figure 3 ). Women with primary PVD $(\mathrm{n}=13)$ had more muscle tenderness $905 \mathrm{~g}$ ( $\mathrm{SD}=527$ ) compared to women with secondary PVD $\mathrm{n}=17$, $1,276 \mathrm{~g}(\mathrm{SD}=692)$, although this difference did not reach statistical significance $(P=0.15)$ (Figure 4$)$.

\section{Discussion}

This proof of concept study suggests differences in the relationship between intercourse pain (dyspareunia) report

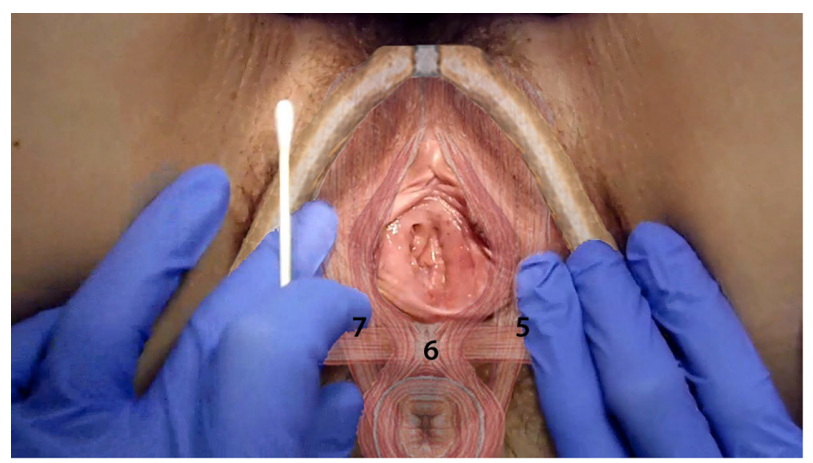

Figure 3 Anatomical location of muscle sites tested.

Notes: Reprinted from The Journal of Pain; 13(9); Zolnoun D, Bair E, Essick G, Gracely R, Goyal V, Maixner W; Reliability and reproducibility of novel methodology for assessment of pressure pain sensitivity in pelvis; pages 910-920; Copyright 2012, with permission from Elsevier. ${ }^{18}$ and mucosal and pelvic muscle sensitivity. The association between intercourse pain report and pelvic muscle pain was both stronger and statistically significant compared to that of the mucosal sensitivity; yet, the association of mucosa sensitivity was moderate overall. Furthermore, women with primary and secondary PVD did not differ on the spectrum of mucosal sensitivity.

As this is one of the first studies to examine clinical correlates to mucosal and muscle sensitivity, ${ }^{8}$ our study has some limitations that should be considered when interpreting our findings. First, muscle assessment involved gradual pressure application with a termination point that could have been as high as 3,000 $\mathrm{g}$ and as low as $500 \mathrm{~g}$. We used a continuous pressure application (at an approximate rate of 500/sec) because the range of pressure pain sensitivity in the pelvic muscles was unknown. As such, the reliability of measures are unlikely to be as high as that described for the conventional approach where a set amount of pressure is applied by the examiner in all the participants. However, a single examiner (DZ) has conducted the exam at one point in time with high within-subject reproducibility (200-300 g difference between successive testing); this limitation is unlikely to significantly alter our findings. Second, we only investigated the relationship between 6 o'clock muscle and the clinical pain report due to a small sample size, the statistical concern about multiple testing, and accepted norms. Third, we used a composite score for lower vestibule averaging pain intensity scores across sites 5, 6, and 7. Because of the small surface area of the vestibule, we anticipated that these sites were equally likely to contribute to experience of pain with intercourse. Detailed discussion of site-specific characteristics of mucosal and muscle pain thresholds, along with the reliability and feasibility of contemporaneous assessment of pelvic muscle and mucosa is described elsewhere. ${ }^{18}$

In this study, muscle site 6 o'clock was noted to be the most sensitive and was therefore used in our analysis. This observation is nearly opposite of what we reported in a recently published study using a far more nuanced methodology.$^{18}$ What is noteworthy is that these two studies were nearly contemporaneous. Specifically, the clinically relevant "bed side" assessment (in the present study) was intended to inform the design of the nuanced protocol. As we described in our methodology paper ${ }^{18}$ the gynecological sensory assessment could significantly alter exam findings. For example, in this study the examiner inadvertently used an inconsistent methodology for exams based on varying clinical scenarios. In women with primary PVD, the examiner tried to minimize emotional aversion and discomfort by using the same index finger (right hand) to examine both 


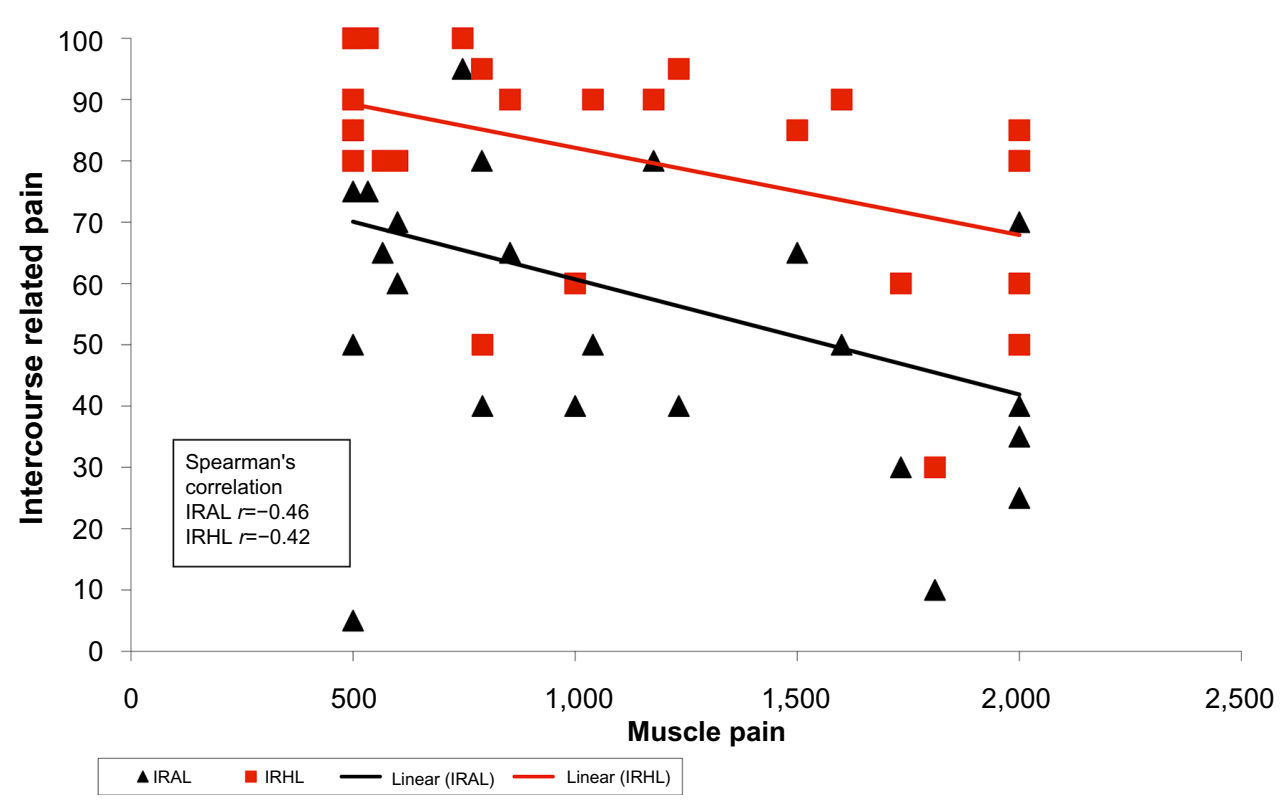

Figure 4 Scatter plot of intercourse pain vs muscle pain detection.

Notes: The black line represents spearman's correlation between average pain report with intercourse and muscle pain detection threshold, while the red line represents the correlation between highest pain with intercourse and muscle pain detection threshold.

Abbreviations: IRAL, average pain with intercourse; IRHL, highest pain with intercourse.

right, left, and mid-perineal position. However, in most women with the secondary subtype, when deemed feasible, the examiner would change hands in order to ensure a more accurate pressure application. The end result was significant variability in muscle tenderness among subgroups, and inconsistent assessment of pelvic muscles, hence fewer women with data on pelvic muscle sensitivity. Nevertheless, it was in the course of this assessment that we made the important observation that the pattern of muscle and pelvic floor sensitivity differed among women with primary and secondary vestibulodynia. Women with primary PVD subtype tended to be consistently more tender at 5 and 7 o'clock muscle position without much tenderness at 6 o'clock position; ${ }^{18}$ secondary subtypes varied from person-to-person though it commonly involved varying degrees of tenderness at 6 o'clock position. Because this study was conducted in a clinical setting with a higher number of women with secondary PVD, the 6 o'clock position emerged as being most sensitive.

We similarly observed challenges in assessing mucosal sensitivity among women with secondary PVD, many of whom were parous. In this subgroup, we could not consistently identify a mucocutaneous junction (Hart's line), and not uncommonly the vulvar mucosa indiscriminately was replaced by vaginal epithelium leading to a patchy perception. For example, a patient may have reported moderate tenderness on the intact mucosa on the left side but no tenderness, or much lower levels of tenderness, on the right side that was replaced by the vaginal epithelium following childbirth. Thus, similar to muscle assessment, mucosal assessment proved to be more challenging than initially assumed. The limited association between clinical pain report and mucosal sensitivity may in part be explained by methodological challenges of assessing mucosa, particularly in parous women.

The limitation of the mucosal exam to reliably predict intercourse pain and differentiate subgroups of women with PVD raises fundamental questions regarding the adequacy of the mucosal sensitivity for classification purposes. Furthermore, the variation of mucosal sensitivity may theoretically result from not only intrinsic mucosal changes, but also from neuromuscular dysfunction and hypertonicity with referred neurogenic sensitivity. Reliability, reproducibility, and the validity of the mucosal assessment are further compounded by yet uncharacterized anatomical variation.

Despite emerging data highlighting the complexity of pain among women with PVD, the diagnosis and classification of patients with PVD continues to be based on one-dimensional mucosal sensitivity. The findings of this study and, and perhaps more importantly, the challenges encountered in the course of this investigation, have enabled us to pilot and describe a more nuanced methodology for multidimensional assessment of pain in the pelvic region that reflects women's report of pain. Further study on the effectiveness of physical therapies such as soft tissue manipulation, myofascial release, dry needling, 
and core strengthening exercises which may address specific sources of muscle pain, and ultimately affecting patient outcomes such as dyspareunia, should be investigated.

\section{Acknowledgments}

This work was in part supported by the NIH-Building Interdisciplinary Research Careers in Women's Health (BIRCWH), K12 HD01441 (Principal Investigator [PI]: Denniz Zolnoun), DE07509 (PI: William Maixner), NS045688 (PI: William Maixner).

\section{Disclosure}

The authors report no conflicts of interest in this work.

\section{References}

1. Harlow BL, Stewart EG. A population-based assessment of chronic unexplained vulvar pain: have we underestimated the prevalence of vulvodynia? J Am Med Women's Assoc. 2003;58(2):82-88.

2. Reed BD, Crawford S, Couper M, Cave C, Haefner HK. Pain at the vulvar vestibule: a web-based survey. J Low Genit Tract Dis. 2004;8(1): 48-57.

3. Goetsch MF. Vulvar vestibulitis: prevalence and historic features in a general gynecologic practice population. Am J Obstet Gynecol. 1991;164(6 Pt 1):1609-1614; discussion 1614-1606.

4. Harlow BL, Wise LA, Stewart EG. Prevalence and predictors of chronic lower genital tract discomfort. Am J Obstet Gynecol. 2001;185(3): $545-550$.

5. Nunns D. Vulval pain syndromes. BJOG. 2000;107(10):1185-1193.

6. Friedrich EG Jr. Vulvar vestibulitis syndrome. J Reprod Med. 1987; 32(2):110-114.

7. Baggish MS, Miklos JR. Vulvar pain syndrome: a review. Obstet Gynecol Surv. 1995;50(8):618-627.

8. Heddini U, Bohm-Starke N, Nilsson KW, Johannesson U. Provoked vestibulodynia - medical factors and comorbidity associated with treatment outcome. J Sex Med. 2012;9(5):1400-1406.

9. Montenegro ML, Mateus-Vasconcelos EC, Rosa e Silva JC, NogueiraAA, Dos Reis FJ, Poli Neto OB. Importance of pelvic muscle tenderness evaluation in women with chronic pelvic pain. Pain Med. 2010;11(2): 224-228.

10. Montenegro ML, Vasconcelos EC, Candido Dos Reis FJ, Nogueira AA, Poli-Neto OB. Physical therapy in the management of women with chronic pelvic pain. Int J Clin Pract. 2008;62(2):263-269.
11. Pukall CF, Payne KA, Binik YM, Khalifé S. Pain measurement in vulvodynia. J Sex Marital Ther. 2003;29 Suppl 1:111-120.

12. Polpeta NC, Giraldo PC, Teatin Juliato CR, Gomes Do Amaral RL, Moreno Linhares I, Romero Leal Passos M. Clinical and therapeutic aspects of vulvodynia: the importance of physical therapy. Minerva Ginecol. 2012;64(5):437-445.

13. Gentilcore-Saulnier E, McLean L, Goldfinger C, Pukall CF, Chamberlain S. Pelvic floor muscle assessment outcomes in women with and without provoked vestibulodynia and the impact of a physical therapy program. J Sex Med. 2010;7(2 Pt 2):1003-1022.

14. Hartmann D, Strauhal MJ, Nelson CA. Treatment of women in the United States with localized, provoked vulvodynia: practice survey of women's health physical therapists. $J$ Reprod Med. 2007;52(1):48-52.

15. Hartmann D. Chronic vulvar pain from a physical therapy perspective. Dermatol Ther. 2010;23(5):505-513.

16. Goldfinger C, Pukall CF, Gentilcore-Saulnier E, McLean L, Chamberlain S. A prospective study of pelvic floor physical therapy: pain and psychosexual outcomes in provoked vestibulodynia. $J$ Sex Med. 2009;6(7):1955-1968.

17. Bachmann GA, Rosen R, Pinn VW, et al. Vulvodynia: a state-of-the-art consensus on definitions, diagnosis and management. J Reprod Med. 2006;51(6):447-456.

18. Zolnoun D, Bair E, Essick G, Gracely R, Goyal V, Maixner W. Reliability and reproducibility of novel methodology for assessment of pressure pain sensitivity in pelvis. J Pain. 2012;13(9):910-920.

19. Zolnoun DA, Rohl J, Moore CG, Perinetti-Liebert C, Lamvu GM, Maixner W. Overlap between orofacial pain and vulvar vestibulitis syndrome. Clin J Pain. 2008;24(3):187-191.

20. Zolnoun D, Park EM, Moore CG, Liebert CA, Tu FF, As-Sanie S. Somatization and psychological distress among women with vulvar vestibulitis syndrome. Int J Gynaecol Obstet. 2008;103(1):38-43.

21. Reeves JL, Jaeger B, Graff-Radford SB. Reliability of the pressure algometer as a measure of myofascial trigger point sensitivity. Pain. 1986;24(3):313-321.

22. Dworkin SF, LeResche L. Research diagnostic criteria for temporomandibular disorders: review, criteria, examinations and specifications, critique. J Craniomandib Disord. 1992;6(4):301-355.

23. Wolfe F, Smythe HA, Yunus MB, et al. The American College of Rheumatology 1990 Criteria for the Classification of Fibromyalgia. Report of the Multicenter Criteria Committee. Arthritis Rheum. 1990; 33(2):160-172.

24. Gracely RH. Evaluation of multi-dimensional pain scales. Pain. 1992;48(3):297-300.
Journal of Pain Research

\section{Publish your work in this journal}

The Journal of Pain Research is an international, peer-reviewed, open access, online journal that welcomes laboratory and clinical findings in the fields of pain research and the prevention and management of pain. Original research, reviews, symposium reports, hypothesis formation and commentaries are all considered for publication.

\section{Dovepress}

The manuscript management system is completely online and includes a very quick and fair peer-review system, which is all easy to use. Visit http://www.dovepress.com/testimonials.php to read real quotes from published authors. 